\title{
FERNAND HAYOT
}

Lattice gas hydrodynamics adopts a microscopic approach to fluid flow. What makes it attractive is its computational simplicity, which includes treatment of complicated boundaries, numerical stability and an obviously parallelizable algorithm.

The aim of my work over the last few years has been to push the microscopic viewpoint into challenging new directions in order to test its ingredients, and better circumscribe its domain of applicability. The problem dealt with mainly is that of the Saffman - Taylor instability. I will review and discuss it in section 2. I mention in section 3. and 4. additional work on flow around a cylinder and on, most recently, turbulent channel flow. Analytical work by my student Don Burgess on bubbles in Hele-Shaw cell flow will be described in section 5. Section 6 alludes to additional work on spiral turbulence and wetting.

\section{Saffman - Taylor Instability}

The Saffman - Taylor instability is the fingering instability which occurs at the interface between two immiscible fluids, when the less viscous one, such as water, pushes the more viscous one, such as oil. It happens in Hele- Shaw cell flow and in porous media. The basic length scale is the width of the cell or a scale much larger than any pore diameter.

It is a macroscopic problem par excellence. The basic ingredients are two typical macroscopic laws, Darcy's and Laplace's. Darcy's law relates fluid velocity to pressure gradient, and Laplace's law introduces surface tension into the expression for interfacial pressure difference. Deriving these laws from the microscopic dynamics of a discrete binary 
gas is undoubtedly a significant step.

In a first paper ${ }^{1}$ we showed how Darcy's law emerges in a two dimensional flow, when the restrictions to the flow caused by pores are mimicled by a low density of scatterers. In two further papers ${ }^{2,3}$ we constructed algorithms for an interface between two immiscible fluids, and studied its properties. The approach taken is semi-macroscopic. The interface is a continuous chain of links, lying along a hexagonal lattice, which deforms under the impact of fluid particles. The two algorithms we developed essentially describe how deformations take place. The crucial point is to ascertain that the interface has the right physical properties. We investigated both static properties establishing Laplace's law, and the fluctuations of the interface in the heat bath provided by the two fluids. In particular we showed that the interface behavior follows that described by a noisy Langevin equation. This point is worth emphasizing, namely we were able to corroborate our numerical results by a series of calculations on interface properties, such as surface tension.

Having established Darcy's law and introduced a successful interface algorithm, we are then able ${ }^{4}$ to attack the problem of the Saffrnan-Taylor instability of fluid flow. In a series of simulations we show how the instability occurs at long wavelength, following the pattern derived from linear stability analysis, and takes the form of a finger penetrating from one fluid into the other. Because of the presence of noise, which is intrinsic to the method, and results from the spatial averaging, the finger itself, however, is unstable and exhibits tip splitting. This occurs at large capillary number, where the phenomenon is also observed in actual experiments. This is a case where a mechanism intrinsic to the algorithmic procedure manifests itself as a physical phenomenon. ${ }^{6}$ The lattice gas thus 
duplicates the real world, with the disadvantage that the presence of noise prevents an in-depth quantitative study.

\section{Cylinder Wake}

We did an analysis ${ }^{6}$ of flow dynamics behind a cylinder, studying first the time development of wake length at low Reynolds numbers, then lift and drag on the cylinder once the von Karman street exists. Dependence of the Strouhal number on the ratio of cylinder diameter to channel width was considered.

Two points emerge: one is the crucial presence - in order to obtain agreement with data- of the special, density dependent, modification of the convective Navier - Stokes term, which arises in lattice gas hydrodynamics. The other is the obvious stability of the lattice gas algorithm, when going from low (several tenths) to high (up to 200) Reynolds numbers, which is not the case of the usual algorithms, which cannot deal with the wake and the von Karman street.

\section{Levy Walk and Turbulent Channel Flow}

A statistical mechanics approach to enhanced diffusion, as it occurs in fully developed turbulence, was recently proposed. The approach is based on so-called Levy walks. Imagine a fluid particle jumping from site to site in a flow. If the mean square displacement is finite, the probability density will ultimately be a gaussian. If it is not, it will tend towards a Levy distribution, A Levy walk is a generalization, where to a Levy distribution for distances is added a completion time for each jump. For a sufficiently slowly falling distribution for distances, one recovers Richardson' law, narnely that the mean square displacement 
increases cubically in time.

My work consists in the implementation of a Levy walk at the microscopic level, namely in lattice gas hydrodynamics. It provides a way to go to effectively much higher Reynolds numbers than the size of the system itself would allow. Lattice gas hydrodynamics lends itself naturally to such an implementation, since particles can be exchanged among lattice sites according to some space and time dependent distribution. The algorithm contains one parameter only, once the relation connecting the number of momentum exchanges to distances is chosen. This parameter is the exponent in the probability distribution for distances. The case considered is that of pressure driven channel flow. The approach provides the equivalent of a closure approximation to the Navier - Stokes equation. The flattened velocity profile, flattened relative to a parabolic one, looked at in scaled variables, corresponds to Reynolds numbers of order several ten thousands, although the one derived from channel width and maximum velocity is much smaller. The shape and magnitude of the turbulent logarithmic velocity profile is also recovered from this microscopic implementation of a Levy walk.

\section{Bubble Motion And Bubble Streams}

This is work done by my student Don Burgess, who is now at the Center for Nonlinear Studies in Los Alamos. In a first paper (with M. Foster) ${ }^{8}$ Burgess investigated three dimensional effects as they manifest themselves in effective boundary conditions for two dimensional Hele - Shaw bubbles. The method used is singular perturtation theory. He computed in particular the pressure drop from the front to the back of the bubble, due to a thin wetting film, and showed the existence of a new scaling region for film thickness 
along the bubble sides. In a second paper (with S. Tanveer) ${ }^{9}$ he presented exact solutions for a steady stream of bubbles in a Hele - Shaw cell, when surface tension is neglected. When surface tension is included, numerical and analytical evidence shows that there is a dramatic increase in bubble speeds when the distance between bubbles becomes of the order of a bubble diameter.

\section{Spiral Turbulence And Wetting}

These works deal with two important nonlinear problems. One ${ }^{10}$ concerns spiral turbulence in Taylor - Couette flow. We proposed for the first time a phase equation for the turbulent spiral which coexists with laminar flow. This is a breakthrough for this subcritical bifurcation, which shows strong hysteretic effects. A second one ${ }^{11}$ deals with wetting of a defect plane in the mean field approximation to a lattice model for two fluids.It shows the existence of a new multicritical point in the relevant phase space.

\section{DISCLAIMER}

\footnotetext{
This report was prepared as an account of work sponsored by an agency of the United States Government. Neither the United States Gnvernment nor any agency thereof, nor any of their employees, makes any warranty, express or implied, or assumes any legal liability or responsibility for the accuracy, completeness, or usefulness of any information, apparatus, product, or process disclosed, or represents that its use would not infringe privately owned rights. Refer. ence herein to any specific commercial product, process, or service by trade na'ne, trademark, manufaturer, or otherwise does not necessarily constitute or imply its endorsement, recommendation, or favoring by the United States Government or any agency thereof. The view's mendation necessarily state or reflect those of the United States Government or any agency thereof.
} 


\section{CIRIYAM JAYAPRAKASH}

The program of elucidating general features of extended, non- equilibriurn systems by studying models using a statistical mechanical approach has been continued fruitfully.

\section{Collective Behavior of a Coupled Map System}

\section{With a Conserved Quantity}

The main motivation for this study ${ }^{12}$ arises from the fact that both past studies and recent investigations in the context of self-organized criticality have emphasized the ramifications of conservation laws for spatial and temporal correlations in nonequilibrium systems. We have carried out an extensive numerical investigation of the behavior of coupled sine maps with a locally conserved variable on a square lattice. We have provided supporting analytic arguments based on the renormalization group for the results given below: 1) By varying the parameters of the model parameters we found two distinct "conservation-induced" routes to chaos: One is a first-order transition from a periodic 2 cycle phase with broken time-translation invariance and long-range, striped, spatial order, to a chaotic, spatially-disordered phase in which time-translation invariance is restored; this transition is accompanied by a discontinuous jump in the maximum Lyapunov exponent, $\lambda_{\max }$. The other is a continuous onset of temporal chaos without any change in the spatial symmetry of the phase, which has striped long-range order on both sides of the transition. In this latter case, $\lambda_{m a x}$ increases continuously from zero as the control parameter, $R$, is increased. In the presence of noise, the numerics show $\lambda_{\max }$ growing linearly, in agreement with simple arguments. We believe that these routes to chaos can occur in a broad class of systems. 2) We have argued analytically that, in the presence 
of noise, spatial and temporal correlations decay algebraically, both in the chaotic and nonchaotic phases, though the arguments for the chaotic phases are heuristic. Numerical evidence (strong for the nonchaotic and weaker for the chaotic phases) has been accumulated to demonstrate the algebraic decay $\left(t^{-1}\right.$ or $t^{-2}$ depending on the model) in time of autocorrelations.

\section{Stability of Temporally Periodic States}

Physical systems that are not driven by time-dependent external forces and described by time-translationally invariant equations of motion can exhibit macroscopic properties varying periodically in time. We have studied ${ }^{13}$ discrete-time, synchronously updated, stochastic systems with short-ranged interactions. We have shown that periodic $k$-cycle states with period $k>2$ are typically metastable losing spatial coherence through the nucleation and growth of droplets. $\mathrm{X}$, have constructed rules that exploit lattice anisotropy to suppress droplet fluctuations and so stabilize higher period cycles. We have also argued for unusually rapidly decaying temporal autocorrelations in such systems.

\section{Generic Nonergodic Behavior in Continuous Systems:}

Probabilistic cellular automata that exhibit non-ergodicity in finite regions of parameter space have been constructed by the Russian mathematician Toom. We have constructed $^{14}$ (partial) differential equations that are nonequilibrium variations of timedependent Ginzburg-Landau models; these are argued to oxhibit analogous behavior. The models incorporate asymmetric terms that have been identified as the crucial ingredient leading to anisotropic domain wall dynamics that in turn causes nonergodicity. We 
have studied these models in continuous time both on a discrete lattice and in a spatial continuum ( $i e$ in a stochastic PDE). By analyzing the motion of domain walls we have analytically demonstrated their anisotropic velocity. The effect of curvature was studied by solving the PDE numerically and verifying that domains of opposite sign are eroded linearly in time.

\section{Characterization of Unstable Periodic Orbits}

\section{In Low-Dimensional Chaotic Attractors and Repellers}

A postdoctoral fellow Dr. Ofer Biham working with me and a student of mine, Wolfgang Wenzel have devised ${ }^{15}$ a numerical technique to determine unstable periodic orbits in the chaotic regime of low- dimensional maps. The clever algorithm consists of deriving the trajectories from a suitably defined Hamiltonian and an associated artificial dynamics whose evolution leads to an extremum of the Hamiltonian that corresponds to an exact periodic orbit of the map. This technique has been applied successfully to the complex Henois ramp ${ }^{16}$; this has also enabled the definition of a new symbolic dynamics which holds for real an' complex periodic orbits. Recently, we have applied ${ }^{17}$ the technique to the standard dissipative map and deduced scaling relations. Another postdoctoral fellow associated with me Mark Kvale and $O$. Biham have applied ${ }^{18}$ this technique to the stadium problem and studied the efficacy of the Gutzwiller trace formula for the quantum behavior.

\section{Ginzburg-Landau Theory}

\section{For Oil-Water-Surfactant Mixtures}

Oil-water-surfactant mixtures exhibit a variety of phases and phas equilibria. Sur- 
factant molecules reduce the bare oil-water interfacial tension leading to the formation of bulk phases with a large density of microscopic oil-water interfaces. We have constructed ${ }^{19}$ a Landau-Ginzburg theory with a scalar order parameter for the oil-water subsystem and a vector for the the surfactant part. A detailed density-functional calculation yields threephase coexistence between oil-rich, water-rich, and microemulsion phases and modulated phases including lamellar, columnar, and cubic phases. The computed structure-factor for water in the microemulsion phase shows a variety of trends in qualitative accord with experiments. 


\section{Published and Submitted Work}

(1) K. Balasubramanian,F. Hayot and W. F. Saam, Phys. Rev. A $\underline{36}$ (1988) 2248.

(2) D. Burgess, F. Hayot and W. F. Saam, Phys. Rev. A $\underline{38}$ (1988) 3589.

(3) D. Burgess, F. Hayot and W. F. Saam, Phys. Rev. A $\underline{39}$ (1989) $46 \tilde{9} 5$.

(4) D. Burgess and F. Hayot, Phys. Rev. A $\underline{40}$ (1989) 5187.

(5) F. Hayot, to appear in Physica D $\underline{47}$.

(6) F. Hayot and M. Raj Lakshmi, Physica D $\underline{40}$ (1989) 415.

(7) F. Hayot, to appear in Phys. Rev. A.

(8) D. Burgess and M. R. Foster, Phys. Fluids.

(9) D. Burgess and S. Tanveer, Phys. Fluids, A 7 (1990) 1105.

(10) J. Hegseth, C. D. Andereck, F. Hayot and Y. Pomeau, Phys. Rev. Lett. 62 (1989) 257.

(11) C. Ebner, F. Hayot and J. Cai, to appear in Phys. Rev. A.

(12) G. Grinstein, Y. He, C. Jayaprakash, and B. Bolker, "Coupled Lattice Maps with a Conserved Quantity" submitted to Phys. Rev. A.

(13) C.H. Bennett, G. Grinstein, Y. He, C. Jayaprakash and D. Mukamel, Phys. Rev. A 41 , $1932(1990)$.

(14) Y. He, C.Jayaprakash, and G. Grinstein, Phys. Rev. A 42, 3348, (1990).

(15) O. Bilam and W. Wenzel, Phys. Rev. Lett. $\underline{63}, 819,(1990)$.

(16) O. Biham and W. Wenzel, Phys. Rev. A $\underline{42}, 4639,(1990)$.

(17) W. Wenzel, O. Biham, and C. Jayaprakash, "Periodic Orbits in the Standard Dissipative Map', submitted to Phys. Rev. A

(18) O. Biham and M. Kvale, "Periodic Orbits in the Stadium Problem", submitted for publi- 
cation.

(19) K. Chen, C. Jayaprakash, R.Pandit, and W. Wenzel, "Microemu/sions: A GinzburgLandau Theory," to be published in Phys. Rev.Lett. (1990).

(20) A. Sinha and C. Jayaprakash, Physica D $\underline{39}, 352$ (1989). 

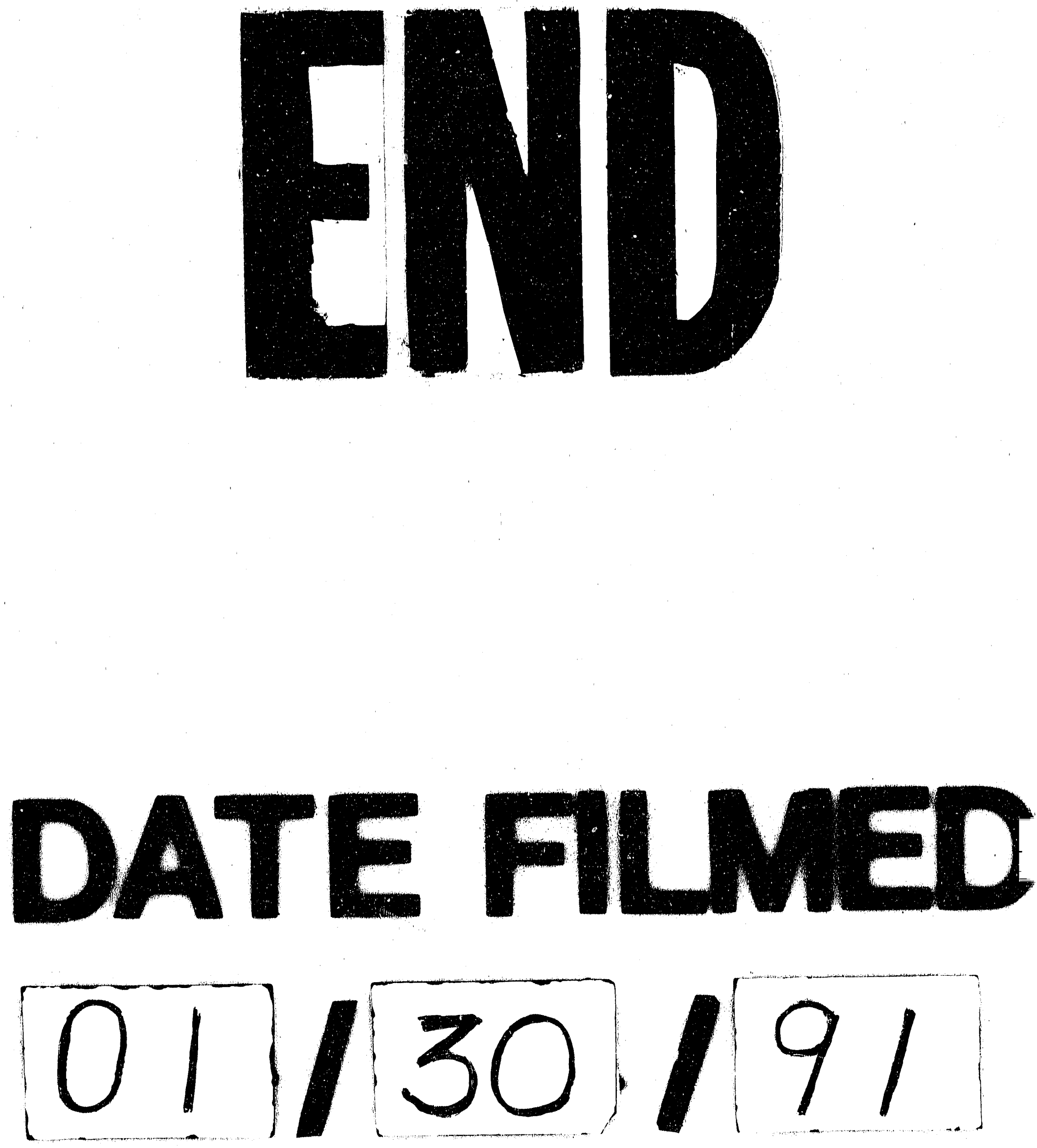